\title{
In vitro and in vivo superior radiosensitizing effect of berbamine for head and neck squamous cell carcinoma
}

This article was published in the following Dove Press journal:

OncoTargets and Therapy

\author{
Hongmei Zhu',* \\ Shu Ruan ${ }^{2, *}$ \\ Feng Jia ${ }^{3, *}$ \\ Jiusheng Chu' \\ Yong Zhu' \\ Yongjiu Huang' \\ Guan Liu ${ }^{4}$
}

'Department of Otolaryngology, Jiangsu Taizhou People's Hospital,

Taizhou 225300, Jiangsu Province, China; ${ }^{2}$ Department of Endocrinology, Yancheng Third Hospital, The Affiliated Hospital of Southeast University Medical College, Yancheng 22400I, Jiangsu Province, China; ${ }^{3}$ Department of Neurosurgery, Yancheng City No I People's Hospital, The Fourth Affiliated Hospital of Nantong Medical College, Yancheng 224005, Jiangsu Province, China; ${ }^{4}$ Department of Radiation Oncology, Zhejiang Cancer Hospital, Hangzhou 310022, Zhejiang Province, China

*These authors contributed equally to this work

Correspondence: Yongjiu Huang

Department of Otolaryngology, Jiangsu

Taizhou People's Hospital, 210 Yinchun

Road, Taizhou 225300, China

$\mathrm{Tel}+865238636$ I5II

Fax +8652386225199

Email 13952645440@I63.com

Guan Liu

Department of Radiation Oncology,

Zhejiang Cancer Hospital, I East Banshan

Road, Hangzhou 310022, China

$\mathrm{Tel}+8657188122222$

Fax +865718 812 2508

Email liuguan@zjcc.org.cn
Background: Berbamine (BBM), one of the bis-benzylisoquinoline products isolated from Berberis amurensis, has been demonstrated for its anticancer effect against leukemia, breast cancer, liver cancer, etc. There are some studies focusing on the chemosensitization effect of BBM. However, there is no report about whether BBM could enhance the anticancer effect of radiation, which made us to explore the possible radiosensitization effect of BBM.

Materials and methods: Here, in vitro cytotoxicity of BBM was evaluated on two kinds of head and neck squamous cancer cell lines. Clonogenic assay was performed to study the radiosensitization effect of BBM. Western blot was utilized to elucidate the possible mechanism underlying the radiosensitization effect.

Results: BBM effectively inhibited the growth of two kinds of cancer cells in a time- and dosedependent manner. Radiation plus BBM led to significantly more reduction of the colony-forming ability of cancer cells when compared with radiation alone. BBM plus radiation led to the most reduction of STAT3 phosphorylation, followed by the significant decrease of the ratio of Bax/ Bcl-2. In vivo study demonstrated that the combinational administration of BBM and radiation generated the most significant tumor-delaying effect among all of the treatment regimens.

Conclusion: We reported, in the current study, the potential role of BBM in not only treating cancer by itself but also offering a promising way to improve the efficacy of radiotherapy by inhibiting the activation of STAT3 and subsequently inducing the apoptosis of cancer.

Keywords: berbamine, radiation, HNSCC, sensitization

\section{Introduction}

Head and neck squamous cell carcinoma (HNSCC) accounts for about half a million of newly diagnosed cases, which is the sixth leading cause of cancer by incidence all over the world. ${ }^{1}$ Radiation therapy is one of the most important ways to treat HNSCC. ${ }^{2} \mathrm{How}-$ ever, the clinical efficacy of radiation therapy is often undermined by the emerging resistance of cancer cells. ${ }^{3,4}$ Although cisplatin and paclitaxel are common radiosensitizers in the clinic, the side effects of concurrent chemoradiation are unavoidable and intolerable for some patients. ${ }^{5}$ For example, compared to patients receiving radiation alone, the incidence of considerable toxicity is much stronger in patients receiving cisplatin-based chemoradiation therapy leading to more morbidity. ${ }^{6}$ Thus, novel radiosensitizers with stronger effect and lower toxicity are always desirable for better treatment.

Recently, traditional Chinese medicine attracts intensive research interests for the reason that most of them possess the activity for various tumors. ${ }^{7-9}$ Berbamine (BBM), one of the bis-benzylisoquinoline products isolated from Berberis amurensis, has been demonstrated for its anticancer effect against several kinds of cancer such as lung 
cancer, liver cancer and prostate cancer. ${ }^{10-13}$ Several studies have reported that BBM effectively inhibits the proliferation of cancer cells including liver cancer, prostate cancer, pancreatic cancer and lung cancer. The possible mechanisms underlying the antitumor effect of BBM include regulating apoptotic proteins. ${ }^{12}$ Moreover, there are some studies reporting that BBM could synergistically enhance the anticancer effect of common chemotherapeutics such as paclitaxel, cisplatin and gemcitabine. ${ }^{14,15}$ However, there is no study focusing on the radiosensitization effect of BBM.

In the current study, we investigated the cytotoxic effect of BBM against HNSCC cells, as well as the apoptotic induction. Then, the radiosensitization effect of BBM was evaluated by clonogenic assay. The expression of related proteins was measured by Western blot analysis to elucidate the possible mechanism underlying the radiosensitization effect. Animal study was performed to evaluate the in vivo radiosensitization effect of $\mathrm{BBM}$.

\section{Materials and methods Materials}

BBM was purchased from Boteshi Biotech Co. Ltd. (Nanjing, China). BBM was dissolved in DMSO as stock solution $(10 \mathrm{mM})$ and diluted with medium before use. Human HNSCC cell line $\mathrm{FaDu}$ (derived from a hypopharyngeal SCC) and $\mathrm{KB}$ (an oral cavity squamous cell carcinoma cell line) were purchased from the Institute of Biochemistry and Cell Biology, Chinese Academy of Sciences (Shanghai, China). Human HNSCC cells were cultured in RPMI-1640 medium supplemented with $10 \% \mathrm{FBS}, 100$ units/mL penicillin $\mathrm{G}$ and $100 \mu \mathrm{g} / \mathrm{mL}$ streptomycin at $37^{\circ} \mathrm{C}$ in a humidified incubator with 5\% $\mathrm{CO}_{2}$. Nude mice (male; nu/nu; 6-8 weeks old with weight about $18 \mathrm{~g}$ ) were obtained from the Model Animal Institute of Nanjing University (Nanjing, China).

\section{Methods}

\section{Cytotoxicity test}

$\mathrm{FaDu}$ and $\mathrm{KB}$ cells were seeded in 96-well plates with a density of 5,000 cells per well and allowed to attach for 24 hours. The cells were then exposed to escalated doses of $\operatorname{BBM}(4,8,16,32,64$ and $128 \mu \mathrm{g} / \mathrm{mL})$ and incubated for 24,36 and 48 hours, respectively. The viability of cells was measured by XTT assay as described in earlier study.

\section{Clonogenic assay and acridine orange (AO)/ethidium} bromide (EB) dual staining

$\mathrm{FaDu}$ and $\mathrm{KB}$ cells were seeded in six-well plates and allowed to grow to $80 \%$ confluence. The cells were treated with $8 \mu \mathrm{g} / \mathrm{mL}$ BBM and irradiated with a dose of 2, 4 and
6 Gy at room temperature. The cells were then immediately trypsinized and seeded in six-well plates at different densities. After 2 weeks, the cells were dyed by crystal violet and the colonies were counted manually to calculate the radiosensitizing effect of different treatments.

$\mathrm{FaDu}$ and $\mathrm{KB}$ cells were seeded in six-well plates at a density of $1 \times 10^{5}$ per well. After 24 hours treatment, the cells were collected, washed and resuspended. Then, the cells were stained with dual fluorescent staining solution containing $3 \mu \mathrm{L}$ of $\mathrm{AO}$ and $3 \mu \mathrm{L}$ of $\mathrm{EB}$ in the dark at $37^{\circ} \mathrm{C}$ for 5 minutes. The morphology of apoptotic tumor cells was observed using a fluorescent microscope (Olympus Corporation, Tokyo, Japan).

\section{Fluorescence activated cell sorting (FACS)}

$\mathrm{FaDu}$ and $\mathrm{KB}$ cells were treated with $\mathrm{BBM}$ and radiation singly or in combination. The dose of BBM was $8 \mu \mathrm{g} / \mathrm{mL}$ and the dose of irradiation was $4 \mathrm{~Gy}$. The apoptosis of FaDu cells was measured by dual staining of annexin V-FITC and propidium iodide (PI) with a commercial kit and analyzed by FACScan flow cytometer (Becton Dickinson, San Jose, CA, USA).

\section{Western blot}

FaDu cells were seeded in petri dishes and grown to $70 \%-80 \%$ confluence. The cells were then exposed to single or combinational treatment of BBM and irradiation. Total proteins were then extracted and subjected to a commercial quantification kit (Pierce Biotechnology, Rockford, IL, USA). Western blot analysis was performed as reported in our previous study. Primary antibodies included anti-STAT3, anti-Bax, anti-Bcl2 and anti-Bcl-xl. Horseradish peroxidaseconjugated secondary antibodies were selected according to the origin of primary antibodies. The expression of proteins was analyzed through the semi-quantification of the bands by image analysis software (Quantity One; Bio-Rad Laboratories Inc., Hercules, CA, USA).

\section{Animal study}

The in vivo animal studies were executed in full compliance with the ARRIVE guidelines (Animal Research: Reporting In Vivo Experiments; the ZJCC Guide for the Care and Use of Laboratory Animals) for cancer study approved by the Animal Care Committee at Zhejiang Cancer Hospital (Hangzhou, People's Republic of China). The density of FaDu cell suspension was adjusted to $1.5 \times 10^{7}$ per $\mathrm{mL} .0 .1 \mathrm{~mL}$ of the suspension with $1.5 \times 10^{6}$ cells was injected subcutaneously into the left axillary space of nude mice. The day when the tumor nodules reached $70-80 \mathrm{~mm}^{3}$ was designated as Day 1 . The mice were randomly divided into four groups: the saline group, BBM group, radiation group and BBM plus radiation group. The dose of BBM 
was $15 \mathrm{mg} / \mathrm{kg}$ while the dose of irradiation was $10 \mathrm{~Gy}$. Mice in the combinational group were treated with i.v. injection of BBM followed by a single irradiation of 10 Gy at the second day. Tumor nodules were measured by a caliber every other day. The volume was calculated with the formula below:

$$
\text { Tumor volume }=1 / 2 \times \mathrm{W} 2 \times \mathrm{L}
$$

where $\mathrm{W}$ represents the width and L represents the length of tumor nodule. Relative tumor volume (RTV) was the ratio of $\mathrm{Vn}$ and V1 (Vn/V1), where Vn is the tumor volume on the corresponding day and V1 is the tumor volume on Day 1. Tumor inhibition rate $(\mathrm{T} / \mathrm{C} \%)$, another derivative indicator to evaluate the antitumor effect of drugs, was calculated as the ratio of Trtv and Crtv (Trtv/Crtv), where Trtv means the RTV of treatment group and Crtv means the RTV of control group. The body weight of mice was also measured every 2 days during the whole experiment.

\section{Statistics}

Data in this study were presented as mean \pm SD. Statistical comparisons were performed by Student's $t$-test or ANOVA. The $P$-value less than 0.05 was accepted as significantly different.

\section{Results}

\section{BBM inhibits the growth of HNSCC cancer cells in a time- and dose- dependent manner}

The in vitro cytotoxicity of BBM was evaluated by 2,3-bis(2-methoxy-4-nitro-5-sulfophenyl)-2H-tetrazolium-5-carbo xanilide (XTT) assay. As shown in Figure 1, BBM effectively inhibited the growth of two kinds of cancer cells in a time- and dose-dependent manner. The IC50 values of BBM against cancer cell line FaDu were 147.7, 39.6 and $14.1 \mu \mathrm{g} / \mathrm{mL}$ for 24 , 36 and 48 hours, respectively. For another kind of cancer cell line KB, the IC50 values were lower than those in cancer cell line FaDu. For example, the IC50 value of BBM for 24 hours incubation in cancer cell line $\mathrm{KB}$ was $81.2 \mu \mathrm{g} / \mathrm{mL}$, which was significantly lower than $147.7 \mu \mathrm{g} / \mathrm{mL}$ in cancer cell line FaDu. Extension of incubation time could partly narrow this kind of discrepancy. The IC50 values of 36 hours on cancer cell line $\mathrm{FaDu}$ and $\mathrm{KB}$ were 33.7 and $39.6 \mu \mathrm{g} / \mathrm{mL}$, respectively, while the IC50 values of 48 hours were 14.1 and $12.9 \mu \mathrm{g} / \mathrm{mL}$, respectively, for each cell line. The results above demonstrate the efficient anticancer effect of BBM on either kind of the two cancer cells.

\section{BBM enhances the radiosensitivity of HNSCC cancer cells}

Then, the apoptosis-inducing effect of BBM plus radiation was investigated by both qualitative and quantitative methods. First, the qualitative evaluation of apoptosis was achieved by $\mathrm{AO} / \mathrm{EB}$ dual staining. The group receiving BBM plus radiation induced more apoptosis than the group receiving radiation alone with more yellow (early apoptosis) and red (late apoptosis) strains (Figure 2A, typical images from FaDu cells). Further, quantification of apoptosis by FACS confirmed the results from AO/EB staining. Both early and late apoptotic cells from the group receiving BBM plus radiation treatment were significantly more than those from the group with radiation treatment alone (Figure $2 \mathrm{~B}$ and $\mathrm{C}$ ).

Next, the radiosensitization effect of BBM on cancer cells was evaluated by the clonogenic assay. As shown in Figure 3,
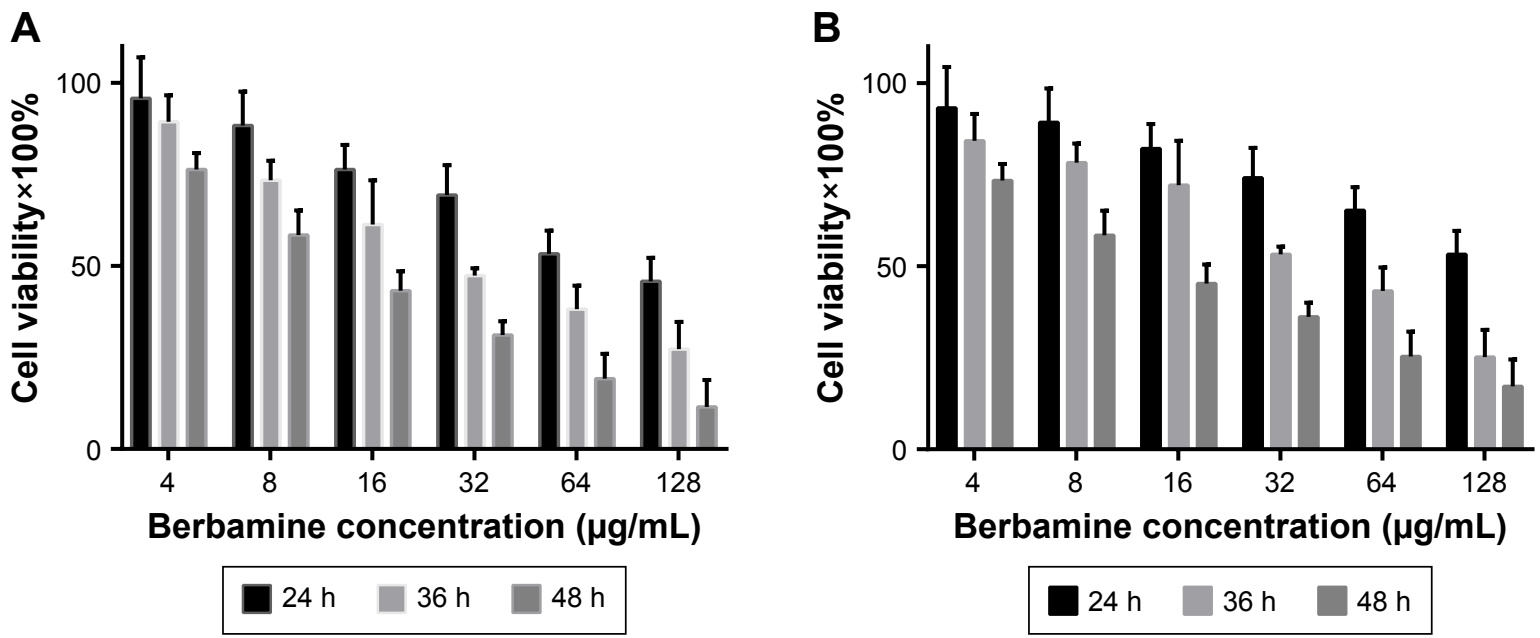

Figure I In vitro cytotoxicity test of BBM was evaluated against two kinds of HNSCC cells. Note: (A) The cytotoxicity of BBM in FaDu cells and (B) the cytotoxicity of BBM in KB cells. Abbreviations: BBM, berbamine; h, hours; HNSCC, head and neck squamous cell carcinoma. 


\section{A}
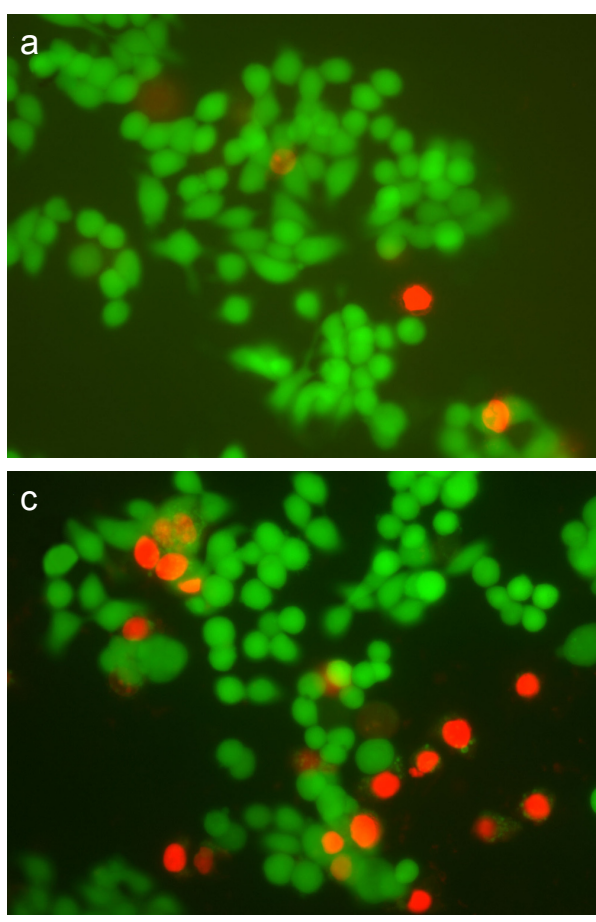

B
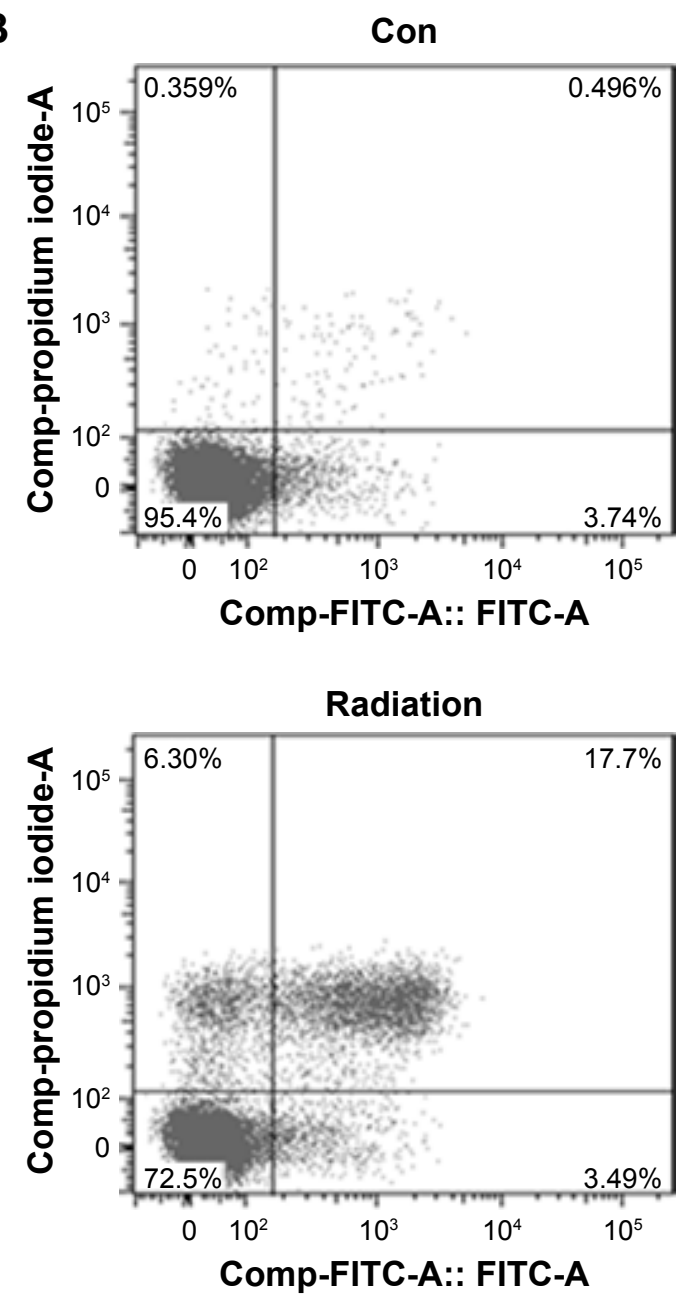
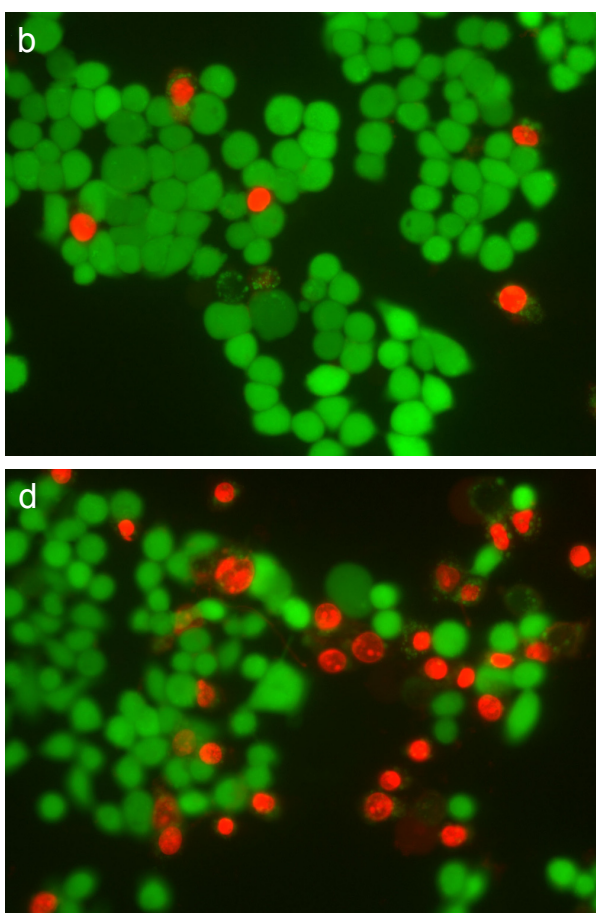

BBM
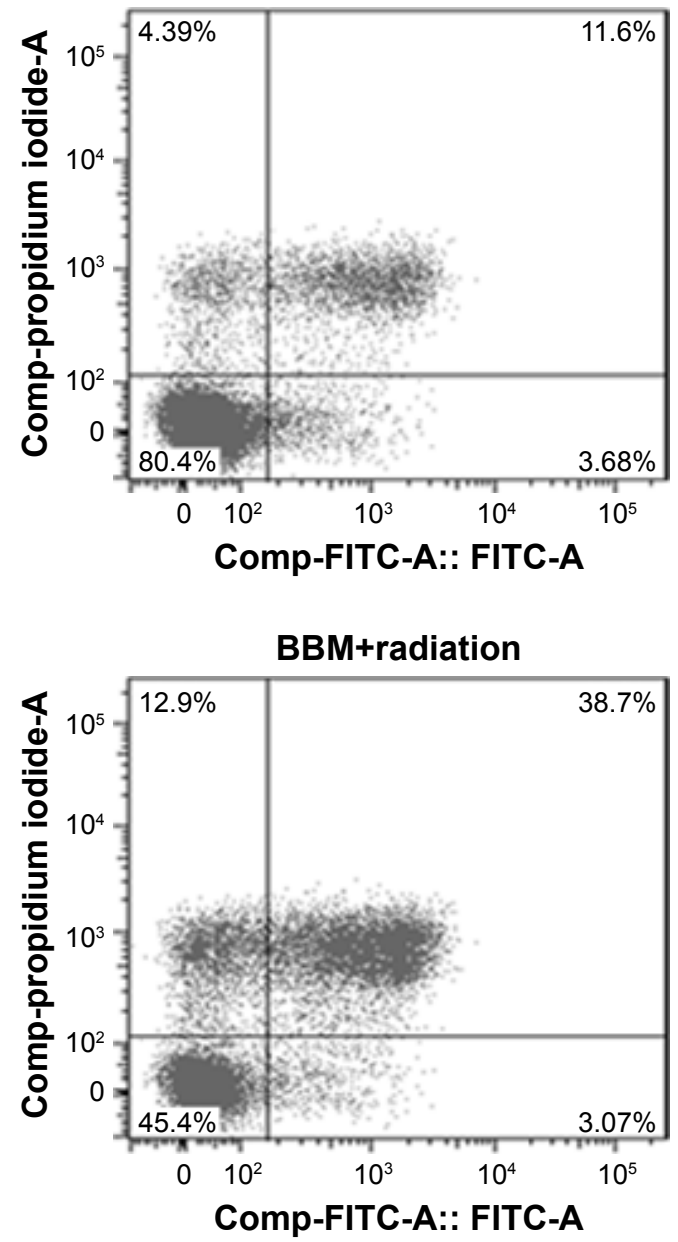

Figure 2 (Continued) 

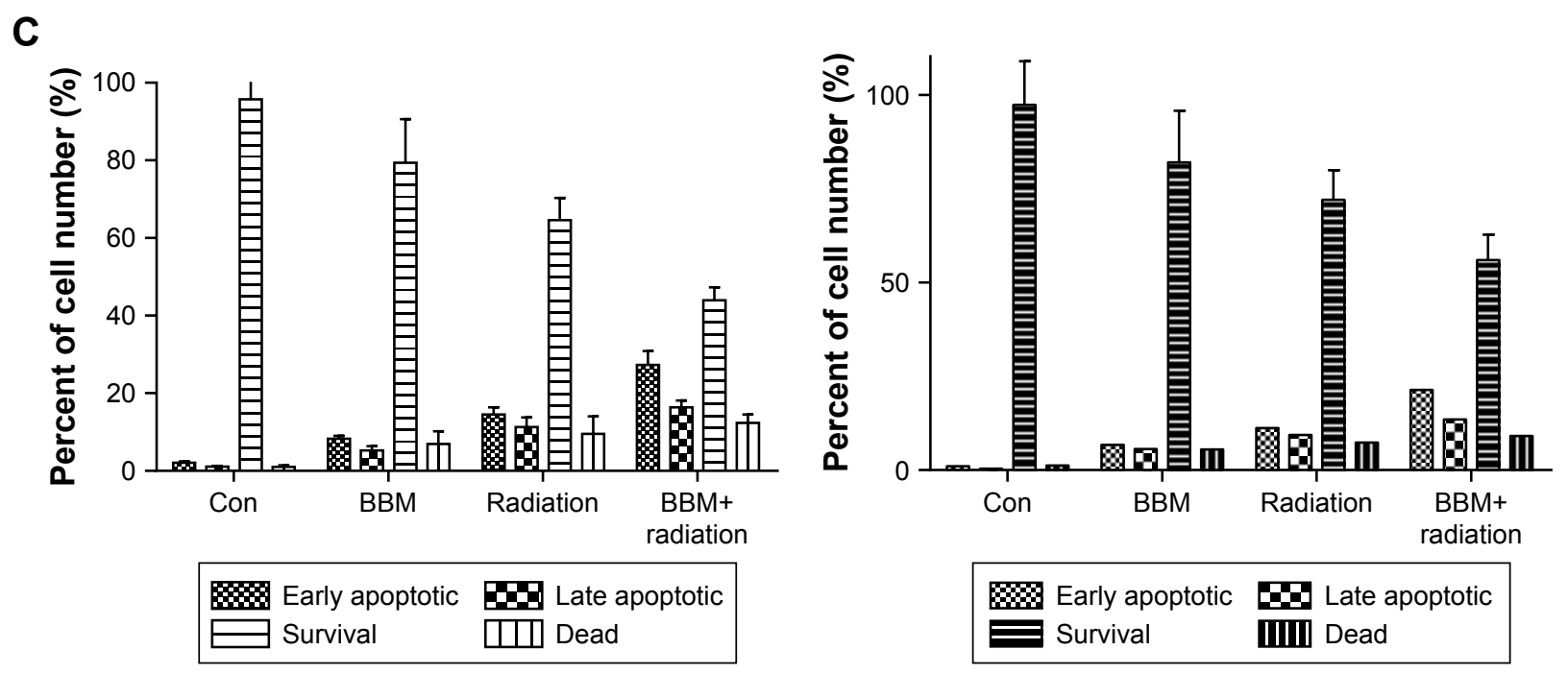

Figure 2 Apoptotic induction of single or combinational administration of BBM and radiation was evaluated in two kinds of HNSCC cells.

Notes: (A) Typical images of FaDu cells exposed to different treatments (a: control; b: BBM; c: radiation; d: BBM plus radiation). Bar represents I0 $\mu$ m. (B) Typical FACS data of FaDu cells exposed to different treatments. (C) Quantification of FACS data in two kinds of cells (the upper panel: FaDu cells; the lower panel: KB cells).

Abbreviations: BBM, berbamine; Con, control; HNSCC, head and neck squamous cell carcinoma.

A

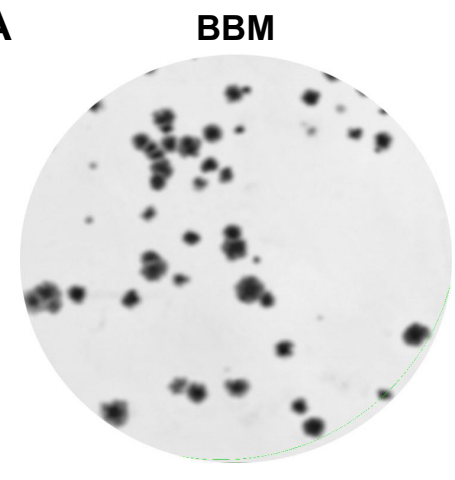

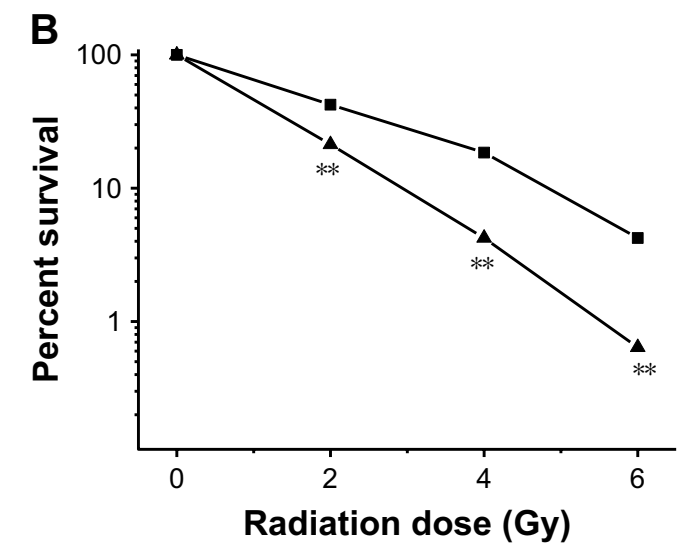

-匹- Radiation -A- BBM+radiation

- Radiation - Ba BMtradiation
Radiation

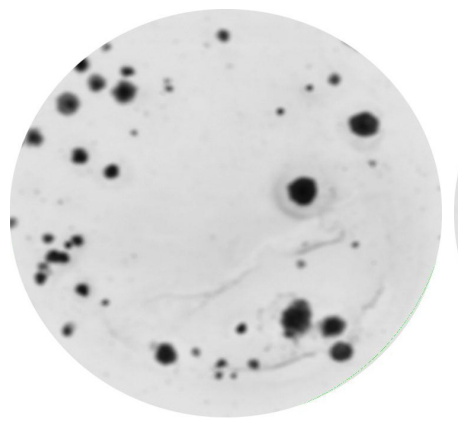

BBM+radiation

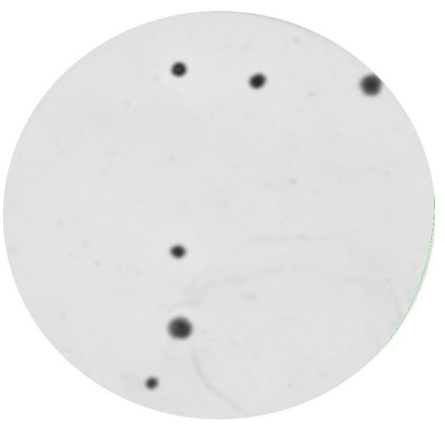

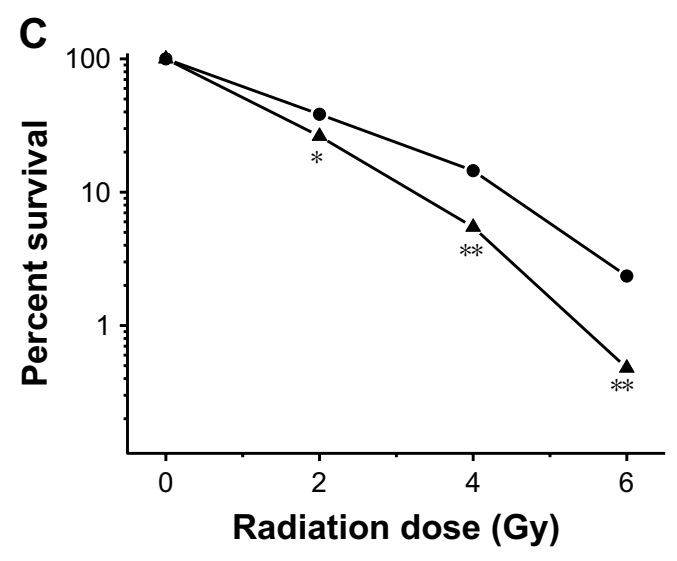

BBM -A- BBM+radiation

Figure 3 Radiosensitization effect of BBM was evaluated in HNSCC cells.

Notes: (A) Typical images of clonogenic assay in FaDu cells. (B) Survival curves of FaDu cells received various treatments indicated. $* P<0.05$; $* * P<0.01$ vs the other group at each radiation dose. (C) Survival curves of $K B$ cells received various treatments indicated. $* P<0.05$; $* * P<0.01$ vs the other group at each radiation dose. Abbreviations: BBM, berbamine; HNSCC, head and neck squamous cell carcinoma. 
both single and combinational application of BBM and radiation therapy effectively inhibited the colony-forming ability of two kinds of cancer cells. Figure 3A shows the typical images of $\mathrm{FaDu}$ cells treated with different regimens. Figure $3 \mathrm{~B}$ and $\mathrm{C}$ shows the survival curves derived from clonogenic assays. Moreover, radiation plus BBM led to significantly more reduction of the colony-forming ability of cancer cells when compared with radiation alone in both cells (Figure 3B and C). There was a significant difference between the percentage of survival in radiation alone and radiation plus BBM groups. As calculated from the curves, the SER values on FaDu and $\mathrm{KB}$ cancer cells were 1.38 and 1.45 , respectively (Figure 3B and C).

\section{Radiosensitization effect of BBM is mediated through the inhibition of $\mathrm{P}$-STAT3 and regulation of the consequent apoptotic proteins}

Western blot analysis was applied to evaluate the possible mechanism of the radiosensitization effect of BBM in FaDu cells. As shown in Figure 4A, either single or combinational administration of BBM and radiation effectively inhibited the phosphorylation of STAT3 proteins. Most importantly, BBM plus radiation led to the most reduction of STAT3 phosphorylation among all of the groups demonstrating that BBM could efficiently sensitize cancer cells to radiation (Figure 4B).
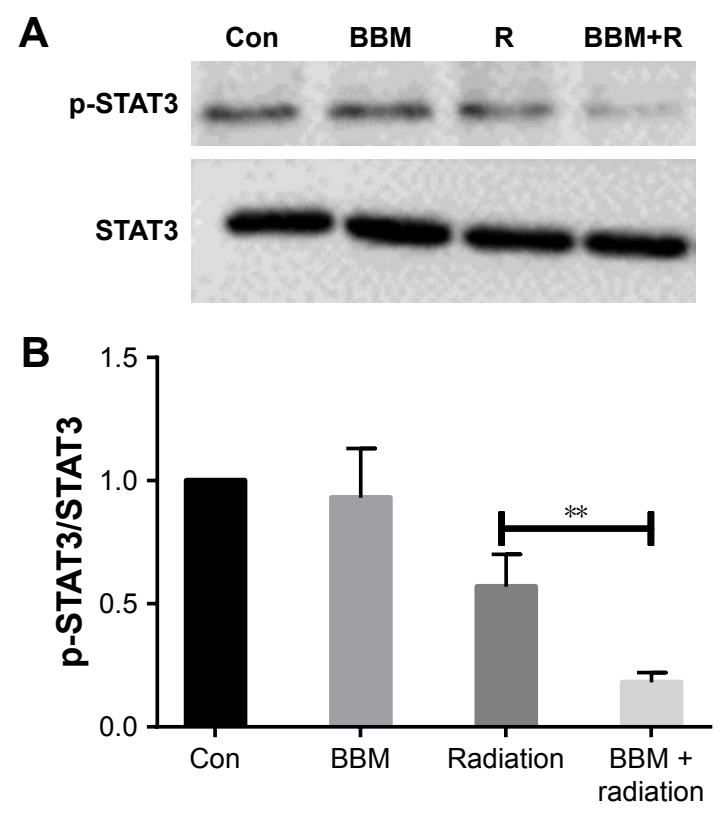

Figure 4 The expression of STAT3 proteins was evaluated in FaDu cells when treated by single or combinational administration of BBM and radiation.

Notes: (A) Typical images of p-STAT3 and STAT3 protein by Western blot. (B) Semi-quantification of different protein expression. $* * P<0.01$.

Abbreviations: BBM, berbamine; Con, control; R, radiation.
Then, we measured the expression of apoptosis-related proteins. Figure 5A shows the change of apoptosis-regulating proteins under different treatments. Either single or combinational treatment of BBM and radiation increased the expression of proapoptotic proteins Bax while decreased the expression of antiapoptotic proteins Bcl-2 and Bcl-xL. Semi-quantification results in Figure 5B indicated that combinational treatment of $\mathrm{BBM}$ and radiation was more efficient to induce apoptosis than a single application of either treatment. Therefore, the combinational application of BBM and radiation significantly lowered the ratio of $\mathrm{Bax} / \mathrm{Bcl}-2$, which demonstrated its superior apoptosis-inducing effect when compared to either of the single treatment.

\section{In vivo radiosensitization effect of BBM in a xenograft model of HNSCC cancer cells}

A xenograft model of cancer cells was established to evaluate the in vivo radiosensitization effect of BBM. The endpoint was reached when the volume of tumor nodule was more than

A
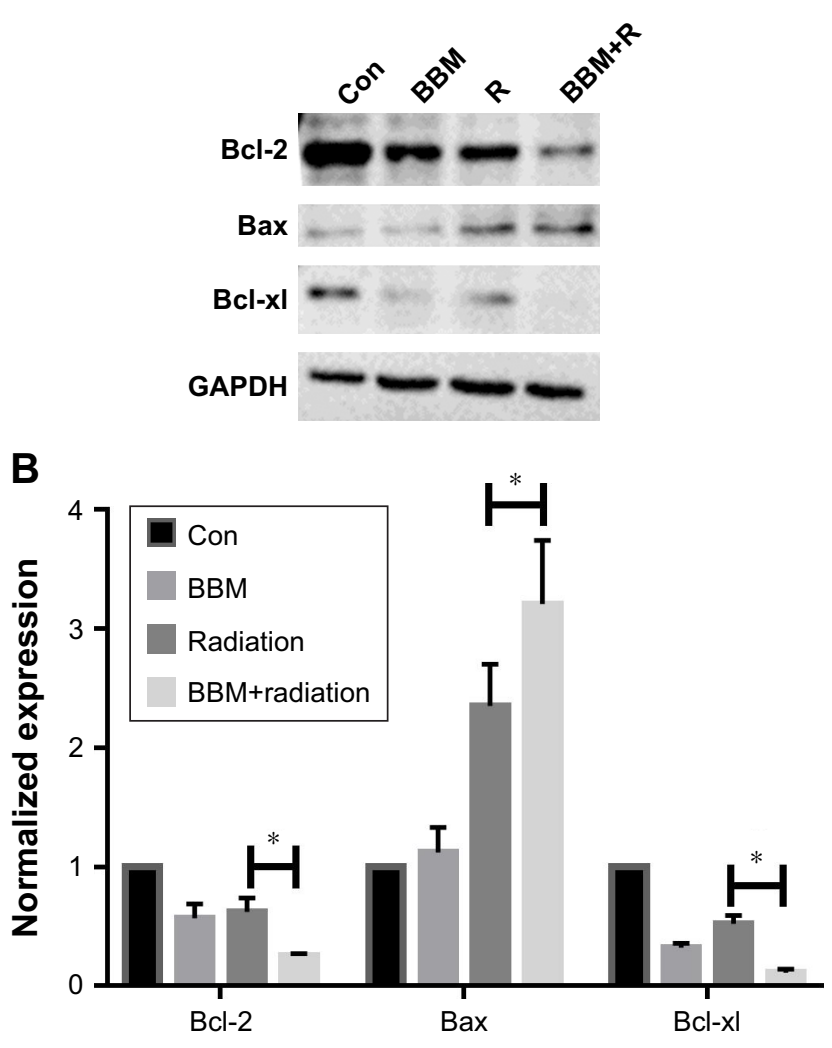

Figure 5 The expression of $\mathrm{Bcl}-2, \mathrm{Bax}$ and $\mathrm{Bcl}-\mathrm{xl}$ proteins was evaluated in $\mathrm{FaDu}$ cells when treated by single or combinational administration of BBM and radiation. Notes: (A) Typical images of different protein expression by Western blot. (B) Semi-quantification of different protein expression. $* P<0.05$.

Abbreviations: BBM, berbamine; Con, control; R, radiation. 
A

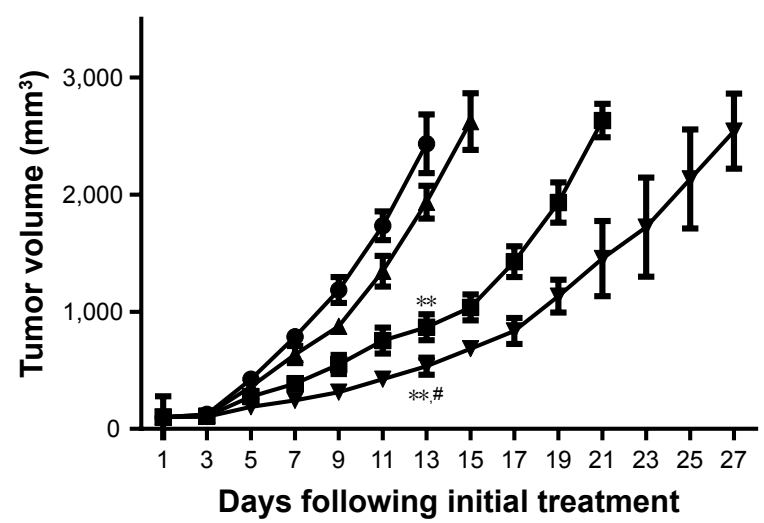

C

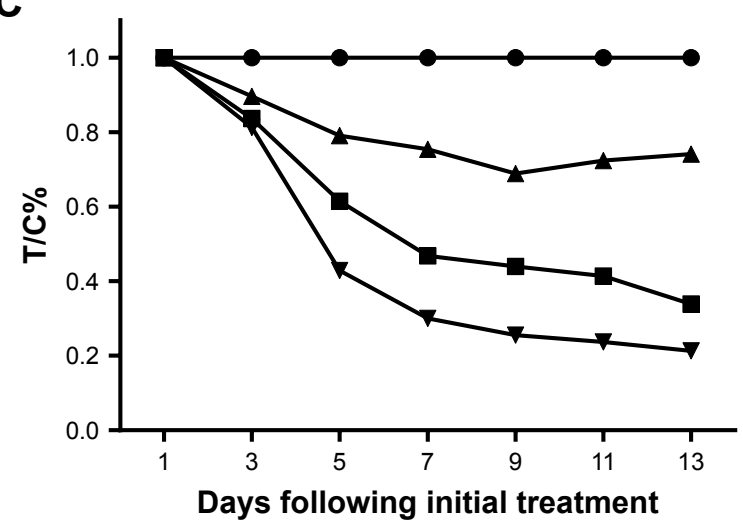

B

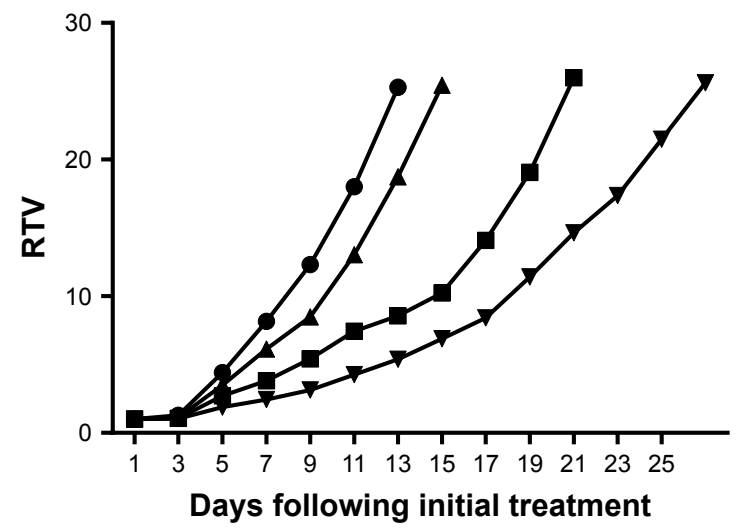

D

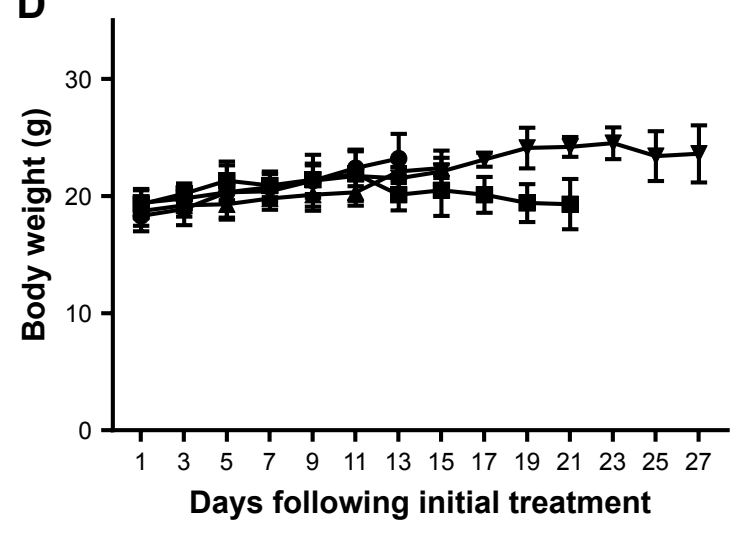

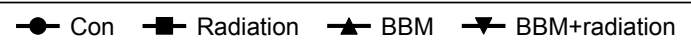

Figure 6 In vivo anticancer effect of BBM plus radiation was evaluated in a xenograft model of FaDu cells.

Notes: Changes in (A) tumor volume, (B) relative tumor volume, (C) therapeutic group/control group (T/C)\% and (D) body weight of mice receiving different therapy treatments. $* * P<0.0$ I vs control; ${ }^{P}<0.05$ vs $B B M$ or radiation group.

Abbreviations: BBM, berbamine; Con, control; RTV, relative tumor volume.

$2,000 \mathrm{~mm}^{3}$. As shown in Figure 6B, RTV of BBM group was 18.7, which was slightly smaller than that of the control group (25.3) on day 13. Radiation treatment alone led to an RTV of 8.56 while RTV of the combinational therapy was 5.74 at that day. After 13 days, mice in the control group were euthanized because the mean tumor volume exceeded 2,000 $\mathrm{mm}^{3}$. Then on day 15 , mice in the BBM group were euthanized due to the same reason. Radiation treatment continued to 23 days before suspension while combinational treatment lasted for 27 days, which was the longest treatment among all the regimens before euthanization. Therefore, either BBM or radiation alone showed slight-to-moderate tumor growth delaying effect against cancer cell xenograft. Radiation alone had a stronger anticancer effect than BBM did. Most importantly, combinational administration of BBM and radiation generated the most significant tumor-delaying effect among all of the treatment regimens (Figure 6A and B).

The derived parameter of tumor volume $(\mathrm{T} / \mathrm{C} \%)$, the tumor inhibition rate of each group, was also calculated to evaluate the tumor-delaying effect (Figure 6C). It is obvious that $\mathrm{T} / \mathrm{C} \%$ of $\mathrm{BBM}$ plus radiation group had been significantly lower than that of other groups since day 5. On day 13 (the end of the control group), T/C $\%$ of the combinational group is $21.2 \%$ while $\mathrm{T} / \mathrm{C} \%$ of $\mathrm{BBM}$ or radiation group was $74.1 \%$ or $33.8 \%$, respectively, which clearly indicated the synergistic in vivo antitumor effect of BBM and radiation.

Animal body weight was measured every other day. Its variations generally define the adverse effect of different treatment regimens. As shown in Figure 6D, there was no significant difference among all the groups, demonstrating that all of the treatments were tolerable by the mice.

\section{Discussion}

As reported previously, $\mathrm{BBM}$ and $\mathrm{BBM}$ derivatives show the potential inhibitory effect on the growth of cancer cells. ${ }^{13,16}$ Some studies report that BBM exerts its anticancer effect through inducing apoptosis, which is mediated by the regulation of various pathways, such as 
JNK/AP-1, Calmodulin-dependent protein kinase II and smad3 pathways. ${ }^{13,17,18}$ Moreover, a literature search in the database indicates that there are some studies focusing on the chemosensitization effect of BBM. BBM and its derivatives could enhance the antitumor effect of paclitaxel, docetaxel and gemcitabine. ${ }^{14,15,19}$ For example, a recent study reported that the synergistic anticancer effect of BBM and paclitaxel was mediated by ROS/Akt pathway, which is the only study focusing on the chemosensitization effect of BBM. ${ }^{14}$ However, there is no report about if BBM could enhance the anticancer effect of radiation, which made us explore the possible radiosensitization effect of BBM.

As reported here, BBM is effective in inhibiting the growth of cancer cells with induction of the apoptosis of cancer cells. ${ }^{12,20}$ Moreover, BBM shows significant potential to sensitize cancer cells to radiation therapy, which suggests that BBM maybe a novel radiosensitizer for the treatment of cancer. Previous studies reported the anticancer effect of BBM against a series of tumors including solid tumors and leukemia. For example, the IC50 of BBM on K562 cells is less than $5 \mu \mathrm{g} / \mathrm{mL} .{ }^{21}$ The IC50 of BBM on solid tumors such as liver cancer is also around $5 \mu \mathrm{g} / \mathrm{mL} \cdot{ }^{13,20}$ Some groups synthesized novel BBM derivatives to improve the antitumor efficacy. ${ }^{16,22}$ In the current study, XTT assay showed that the IC50 of BBM on cancer cells is around $8.3 \mu \mathrm{g} / \mathrm{mL}$, which is in accordance with previous studies. One of the most important findings in the present study is the radiosensitization effect of BBM. Both clonogenic assay and apoptosis detection revealed the efficacy of BBM in sensitizing cancer cells to radiation. The SER values were 1.38 and 1.45 , respectively, on two kinds of cancer cells, which demonstrated a satisfied radiosensitization effect of BBM.

Possible mechanisms underlying the radiosensitization effect may include the inhibition of the phosphorylation of STAT3 and the consequent activation of apoptosis. As reported in earlier studies, constantly enhanced activation of STAT3 represents poor prognosis with radioresistance in patients who suffered from a series of solid tumors. ${ }^{23,24}$ As shown here, the effective inhibition of STAT3 expression by BBM has led to a significant improvement of radiation therapeutic index, which was proved by the results from clonogenic assays and Western blot analysis. Thus, results from the current study provided a novel mechanism for the radiosensitization effect of BBM. STAT3 as a key factor in the development of radioresistance could be significantly inactivated by BBM, which consequently led to the induction of apoptosis and enhanced the efficacy of radiotherapy. ${ }^{25-27}$
It is reported that STAT proteins, a group of transcription factors, which are constantly activated in a series of tumors, are closely related to tumor regression..$^{23,24} \mathrm{~A}$ recent study demonstrates that NSCLC patients with lower expression of STAT3 have longer overall survival than those with high STAT3 expression, which means STAT3 maybe a potential target for cancer therapy. ${ }^{24}$ Moreover, several studies have demonstrated that STAT3 might be a potential target to predict chemo- and radioresistance. ${ }^{28-30}$ Radioresistance is closely associated with the enhanced activation of STAT3, as well as its downstream target genes, such as survivin. On the contrary, inhibition of STAT3 activation is effective to sensitize cancer cells to radiation therapy. Therefore, BBM shows the potential to be a novel radiosensitizer with lower toxicity.

Future studies will be focused on the extensive exploration of the way to optimize the parameters of radiosensitization of BBM. Planned modifications are ongoing in the author's lab to try to further elucidate the synergistic mechanism between BBM and radiotherapy. In addition, screening tests are under active consideration to identify novel derivatives of BBM or other small molecules from herbal medicine with radiosensitizing effect.

\section{Conclusion}

We reported, in the current study, the potential role of BBM in not only treating cancer by itself but also offering a promising way to improve the efficacy of radiotherapy by inhibiting the activation of STAT3 and subsequently inducing the apoptosis of cancer. Moreover, novel derivatives from reasonable modification of BBM may provide a feasible way to enhance the effect of radiation therapy. Additionally, since other kinds of traditional Chinese medicine also possess antitumor effects, they could be potential drug sensitizers for the application of radiation therapy. It is undoubted, however, that the development of traditional Chinese medicine as drug sensitizers warrants more intensive research in order to evaluate the feasibility and advantages of clinical applications.

\section{Acknowledgment}

This work was supported by Project of Jiangsu Administration of Traditional Chinese Medicine of China (YB2017074).

\section{Author contributions}

SR, YH and GL conceived and designed the experiments; $\mathrm{HZ}$ and FJ performed the experiments; JC, YZ and YH analyzed the data; SR and GL contributed reagents/materials/ analysis tools; HZ, SR and GL wrote the paper. All authors 
contributed to data analysis, drafting and revising the article, gave final approval of the version to be published, and agree to be accountable for all aspects of the work.

\section{Disclosure}

The authors report no conflicts of interest in this work.

\section{References}

1. Siegel RL, Miller KD, Jemal A. Cancer statistics, 2018. CA Cancer J Clin. 2018;68(1):7-30.

2. Bourhis J, Lapeyre M, Tortochaux J, et al. Phase III randomized trial of very accelerated radiation therapy compared with conventional radiation therapy in squamous cell head and neck cancer: a GORTEC trial. J Clin Oncol. 2006;24(18):2873-2878.

3. Baumann R, Depping R, Delaperriere M, Dunst J. Targeting hypoxia to overcome radiation resistance in head \& neck cancers: real challenge or clinical fairytale? Expert Rev Anticancer Ther. 2016;16(7):751-758.

4. Bertrand G, Maalouf M, Boivin A, et al. Targeting head and neck cancer stem cells to overcome resistance to photon and carbon ion radiation. Stem Cell Rev. 2014;10(1):114-126.

5. Kitamoto Y, Akimoto T, Ishikawa H, et al. Acute toxicity and preliminary clinical outcomes of concurrent radiation therapy and weekly docetaxel and daily cisplatin for head and neck cancer. Jpn J Clin Oncol. 2005;35(11):639-644.

6. Simpson DR, Song WY, Moiseenko V, et al. Normal tissue complication probability analysis of acute gastrointestinal toxicity in cervical cancer patients undergoing intensity modulated radiation therapy and concurrent cisplatin. Int J Radiat Oncol Biol Phys. 2012;83(1):e81-e86.

7. Li X, Lu X, Xu H, et al. Paclitaxel/tetrandrine coloaded nanoparticles effectively promote the apoptosis of gastric cancer cells based on "oxidation therapy". Mol Pharm. 2012;9(2):222-229.

8. Denham JW, Abbott RL. Concurrent cisplatin, infusional fluorouracil, and conventionally fractionated radiation therapy in head and neck cancer: dose-limiting mucosal toxicity. J Clin Oncol. 1991;9(3):458-463.

9. Wang TH, Wan JY, Gong X, Li HZ, Cheng Y. Tetrandrine enhances cytotoxicity of cisplatin in human drug-resistant esophageal squamous carcinoma cells by inhibition of multidrug resistance-associated protein 1. Oncol Rep. 2012;28(5):1681-1686.

10. Duan H, Luan J, Liu Q, Yagasaki K, Zhang G. Suppression of human lung cancer cell growth and migration by berbamine. Cytotechnology. 2010;62(4):341-348.

11. Hou ZB, Lu KJ, Wu XL, Chen C, Huang XE, Yin HT. In vitro and in vivo antitumor evaluation of berbamine for lung cancer treatment. Asian Pac J Cancer Prev. 2014;15(4):1767-1769.

12. Zhao Y, Lv JJ, Chen J, et al. Berbamine inhibited the growth of prostate cancer cells in vivo and in vitro via triggering intrinsic pathway of apoptosis. Prostate Cancer Prostatic Dis. 2016;19(4):358-366.

13. Meng Z, Li T, Ma X, et al. Berbamine inhibits the growth of liver cancer cells and cancer-initiating cells by targeting $\mathrm{Ca}^{2+} /$ calmodulin-dependent protein kinase II. Mol Cancer Ther. 2013;12(10):2067-2077.

14. Jia F, Ruan S, Liu N, Fu L. Synergistic Antitumor Effects of Berbamine and Paclitaxel through ROS/Akt Pathway in Glioma Cells. Evid Based Complement Alternat Med. 2017;2017:8.

OncoTargets and Therapy

\section{Publish your work in this journal}

OncoTargets and Therapy is an international, peer-reviewed, open access journal focusing on the pathological basis of all cancers, potential targets for therapy and treatment protocols employed to improve the management of cancer patients. The journal also focuses on the impact of management programs and new therapeutic agents and protocols on

Submit your manuscript here: http://www.dovepress.com/oncotargets-and-therapy-journal
15. Jin $\mathrm{X}, \mathrm{Wu} \mathrm{Y}$. Berbamine enhances the antineoplastic activity of gemcitabine in pancreatic cancer cells by activating transforming growth factor-3/Smad signaling. Anat Rec (Hoboken). 2014;297(5):802-809.

16. Yang F, Nam S, Brown CE, et al. A novel berbamine derivative inhibits cell viability and induces apoptosis in cancer stem-like cells of human glioblastoma, via up-regulation of miRNA-4284 and JNK/AP-1 signaling. PLoS One. 2014;9(4):e94443.

17. Liang Y, Qiu X, Xu RZ, Zhao XY. Berbamine inhibits proliferation and induces apoptosis of KU812 cells by increasing Smad3 activity. J Zhejiang Univ Sci B. 2011;12(7):568-574.

18. Liang Y, He X, Li X, et al. 4-Chlorbenzoyl Berbamine, a Novel Derivative of the Natural Product Berbamine, Potently Inhibits the Growth of Human Myeloma Cells by Modulating the NF- $\mathrm{KB}$ and JNK Signalling Pathways. Cancer Invest. 2016;34(10):496-505.

19. Vidal L, Ben Aharon I, Limon D, Cohen E, Popovtzer A. Role of Induction Chemotherapy Prior to Chemoradiation in Head and Neck Squamous Cell Cancer-Systematic Review and Meta-analysis. Cancer J. 2017;23(2):79-83.

20. Cao Y, Cao J, Yu B, et al. Berbamine induces SMMC-7721 cell apoptosis via upregulating p53, downregulating survivin expression and activating mitochondria signaling pathway. Exp Ther Med. 2018; 15(2):1894-1901.

21. Wei YL, Liang Y, Xu L, Zhao XY. The antiproliferation effect of berbamine on k562 resistant cells by inhibiting NF-kappaB pathway. Anat Rec (Hoboken). 2009;292(7):945-950.

22. Yang F, Nam S, Zhao R, et al. A novel synthetic derivative of the natural product berbamine inhibits cell viability and induces apoptosis of human osteosarcoma cells, associated with activation of JNK/AP-1 signaling. Cancer Biol Ther. 2013;14(11):1024-1031.

23. Torres-Roca JF, Desilvio M, Mora LB, et al. Activated STAT3 as a correlate of distant metastasis in prostate cancer: a secondary analysis of Radiation Therapy Oncology Group 86-10. Urology. 2007;69(3): 505-509.

24. Yin ZJ, Jin FG, Liu TG, Fu EQ, Xie YH, Sun RL. Overexpression of STAT3 potentiates growth, survival, and radioresistance of non-smallcell lung cancer (NSCLC) cells. J Surg Res. 2011;171(2):675-683.

25. Lau J, Ilkhanizadeh S, Wang S, et al. STAT3 Blockade Inhibits Radiation-Induced Malignant Progression in Glioma. Cancer Res. 2015; 75(20):4302-4311.

26. Li X, Yu Z, Li Y, et al. The tumor suppressor miR-124 inhibits cell proliferation by targeting STAT3 and functions as a prognostic marker for postoperative NSCLC patients. Int J Oncol. 2015;46(2):798-808.

27. Gao L, Li FS, Chen XH, et al. Radiation induces phosphorylation of STAT3 in a dose- and time-dependent manner. Asian Pac J Cancer Prev. 2014;15(15):6161-6164.

28. Bu X, Zhao C, Wang W, Zhang N. GRIM-19 inhibits the STAT3 signaling pathway and sensitizes gastric cancer cells to radiation. Gene. 2013;512(2):198-205.

29. Otero DC, Poli V, David M, Rickert RC. Cutting edge: inherent and acquired resistance to radiation-induced apoptosis in B cells: a pivotal role for STAT3. J Immunol. 2006;177(10):6593-6597.

30. Zang C, Liu X, Li B, et al. IL-6/STAT3/TWIST inhibition reverses ionizing radiation-induced EMT and radioresistance in esophageal squamous carcinoma. Oncotarget. 2017;8(7):11228-11238.

patient perspectives such as quality of life, adherence and satisfaction. The manuscript management system is completely online and includes a very quick and fair peer-review system, which is all easy to use. Visit http://www.dovepress.com/testimonials.php to read real quotes from published authors. 